\title{
Progressive Pontine-Medullary Dysfunction Leads to REM Sleep Behavior Disorder Symptoms in a Chronic Model of Parkinson's Disease
}

\author{
Lida Du' \\ Linhao $X u^{1,2}$ \\ Tuo Liang' \\ Yun-Kwok Wing (iD ${ }^{3}$ \\ Ya Ke $\mathrm{K}^{\mathrm{l,4}}$ \\ Wing-Ho Yung ${ }^{1,4}$ \\ 'School of Biomedical Sciences, Faculty of \\ Medicine, The Chinese University of \\ Hong Kong, Hong Kong, People's \\ Republic of China; ${ }^{2}$ Department of \\ Cardiology, Affiliated Hangzhou First \\ People's Hospital, Zhejiang University \\ School of Medicine, Hangzhou, People's \\ Republic of China; ${ }^{3}$ Department of \\ Psychiatry, Faculty of Medicine, The \\ Chinese University of Hong Kong, \\ Hong Kong, People's Republic of China; \\ ${ }^{4}$ Gerald Choa Neuroscience Centre, The \\ Chinese University of Hong Kong, \\ Hong Kong, People's Republic of China
}

Correspondence: Wing-Ho Yung; Ya Ke School of Biomedical Sciences, Faculty of Medicine, The Chinese University of Hong Kong, Shatin, Hong Kong, People's Republic of China

Tel +852-39436880; +852-39436780

Fax +852-26035I23

Email whyung@cuhk.edu.hk;

yake@cuhk.edu.hk
Background: Clinical observations reveal that rapid eye movement (REM) sleep behavior disorder (RBD) often develops prior to alpha-synucleinopathies including Parkinson's disease (PD). However, a causal relationship between alpha-synucleinopathy and Parkinsonian neurodegeneration has not been delineated.

Methods: Rats were chronically treated with rotenone and EEG and EMG signals were recorded for analysis of sleep behavior, assisted by video recording of body movements. $\mathrm{C}$-fos expression and TUNEL staining were used to assess neuronal activation and apoptosis, respectively. Chemogenetic manipulation of brain stem nuclei was conducted to ameliorate RBD symptoms in rotenone-treated rats.

Results: Rats chronically exposed to rotenone exhibited progressive RBD features, from EEG slowing to REM sleep motor behavior and NREM muscle activities. Temporally, these phenomena correlated well with progressive alpha-synuclein aggregation and neuronal apoptosis in the sublaterodorsal tegmental nucleus (SLD) and gigantocellular ventricular reticular nucleus in the brainstem. Chemogenetic activation of glutamatergic neurons in SLD alleviated RBD symptoms in the rotenone model.

Conclusion: Taken together, these results are consistent with a progressive degeneration in the REM sleep promoting and atonia circuit in early Parkinsonism that underlies the emergence of RBD symptoms, and demonstrate that the rotenone model is useful for further studies into RBD and its relationship to PD.

Keywords: Parkinson's disease, REM sleep behavior disorder, neurodegeneration, parasomnia, early biomarker

\section{Introduction}

REM sleep behavior disorder (RBD) is a neurobiological parasomnia that is characterized by elaborate and often violent motor behaviors during REM sleep. ${ }^{1} \mathrm{RBD}$ is regarded as a public health concern because it affects $0.4-0.5 \%$ of the general population. ${ }^{2,3}$ It is commonly diagnosed via dream enactment and is always associated with other sleep disturbances, eg, sleep fragmentation, non-REM (NREM) sleep behaviors and reduced REM sleep quality. ${ }^{4,5}$ It is believed that patients have acquired major RBD symptoms when the normal inhibition of spinal motor neurons underlying muscle atonia during REM sleep is lost. ${ }^{6}$ Intriguingly, RBD has a high co-morbidity with neurodegenerative symptoms, ${ }^{7}$ and a large proportion of patients eventually develop neurodegenerative diseases. Emerging data suggest that the development of neurodegeneration in RBD patients is enhanced by the presence of other biomarkers, such as anosmia, impaired 
color vision and neuroimaging features, ${ }^{8-11}$ but RBD is by far the strongest clinical predictor for the onset of neurodegenerative diseases. $^{12,13}$

Although the mechanism of the generation of REM sleep and associated muscle atonia is not fully understood, some previous studies have suggested that REM sleep muscle atonia is triggered by the sublaterodorsal nucleus (SLD) in the pontine tegmentum. $^{14-16}$ As a REM-on brain region, SLD sends ascending projections to intralaminar thalamus mediating cortical activation characteristics of REM sleep. ${ }^{17}$ At the same time, neuronal subpopulation in SLD directly activates downstream medullary GABAergic neurons during REM sleep. ${ }^{18,19}$ Inhibitory GABAergic or glycinergic projections from the ventral medulla in turn inhibit REM-off regions and produce REM sleep muscle atonia by inhibiting spinal motor neurons. $^{20,21}$

Clinical observations and evidence revealed that RBD has a tighter link with synucleinopathies compared with other neurodegenerative disorders. ${ }^{22,23}$ Thus, the major neurodegenerative diseases associated with RBD are characterized by alpha-synucleinopathies, including Parkinson's disease (PD), multiple system atrophy (MSA) and dementia with Lewy bodies (DLB). ${ }^{13,24,25}$ There is evidence that alpha-synuclein may impact the brainstem sleep circuit that controls REM sleep during neurodegeneration. ${ }^{14,26,27}$ Consistent with this notion, REM sleep atonia maintaining pathway can be substantially affected by alpha synucleinopathy, but not tauopathy. ${ }^{28,29}$ Despite these observations, the intrinsic link between RBD and alpha-synuclein aggression remains elusive. It is envisioned that the identification of early-stage alpha-synuclein neurodegeneration would allow for better elucidation of the underlying pathogenic processes and neural pathways of RBD, and also exploration of early protective intervention.

Alpha-synuclein is a well-known pathological hallmark of $\mathrm{PD},{ }^{30}$ and affects various brainstem structures, including medulla, pontine tegmentum and midbrain. ${ }^{31}$ The impact of synucleinopathy on brainstem REM sleep controlling circuitry has been implicated in the pathogenesis of the disorder. ${ }^{32}$ In the present study, to gain insight into the mechanistic relationship between alpha-synuclein accumulation and RBD, we made use of a chronic model of PD based on prolonged administration of rotenone that has been reported to generate features of PD including the inclusion of alphasynuclein. ${ }^{33,34}$ Specifically, we examined the emergence and progression of RBD-like sleep behavioral events and other common RBD symptoms in rotenone-administered rats. We also interrogated the involvement of specific brainstem nuclei with chemogenetic strategies. Our results strongly support a causal link between alpha-synuclein accumulation and the emergence of RBD, and show that the rotenone model is a useful model that can recapitulate the features of $\mathrm{RBD}$, thus providing a foundation for further studies into $\mathrm{RBD}$ and its relationship to neurodegenerative diseases.

\section{Materials and Methods}

\section{Animal Model and Treatment}

Rotenone was dissolved in sunflower oil and stocked in darkness. SD rats were ordered in pairs, with one assigned to the rotenone treatment group and one in the control group. $2.5 \mathrm{mg} / \mathrm{kg}$ dosages of rotenone was administered to the modeling group i.p. for 30 consecutive days or until the appearance of motor deficits. During the same period, control subjects were housed together and received the same daily volume of solvents. Subjects will receive the above treatments with ad libitum access to food/water. Body weight will be recorded daily.

\section{Surgical Procedures for Electroencephalography and Electromyography}

Rats were anaesthetized with $4 \%$ chloral hydrate $(40 \mathrm{mg} / \mathrm{kg})$ and placed in a stereotaxic apparatus. Two holes were drilled into the skull's frontal-parietal region. A dedicated telemetric transmitter connected to bipolar EEG and EMG electrodes to measure and transmit EEG \& EMG signals at certain frequencies (Data Science International, USA) was implanted over the frontal parietal region and placed above meninges. Bipolar EEG electrodes were placed over the left and right frontal cortex $(-1.0 \mathrm{~mm}$ antero-posterior $(\mathrm{A} / \mathrm{P}), \pm 1.5 \mathrm{~mm}$ mediallateral $(\mathrm{M} / \mathrm{L})$ from bregma) for frontal and parietal EEG recordings. Electromyogram (EMG) signals were acquired by bilateral electrodes inserted into the neck extensor muscles. Transmitters were mounted subcutaneously on the back of the animal.

\section{Electroencephalography and Electromyography Recording}

Sleep recordings were performed in home cages and under light phase of 08:00-20:00 and dark phase of 20:00-08:00 (+1 day). The transmitters could be switched on/off by touching the animal with a magnet. The EEG and EMG rhythms were consecutively recorded by the Dataquest A. R.T. software (Data Science International, USA), and some other parameters such as body temperature and moving average were also included in the data file. Each 
recording session typically lasted for 24 hours to record complete circadian rhythm.

\section{EEG/EMG Analyses and Sleep Scoring}

EEG/EMG analyses and sleep scoring methods followed those reported in the literature. ${ }^{16,35,36}$ In brief, all EEG/ EMG recordings were split to consecutive $10 \mathrm{~s}$ segments, and each segment was categorized as wakefulness, REM sleep and non-REM (NREM) sleep. The EEG power spectra were computed for each segment in the frequency range of 1 to $25 \mathrm{~Hz}$ by fast Fourier transformation. Wakefulness is defined by desynchronized EEG and absence of tonic EMG. NREM sleep is defined by synchronized EEG with high-amplitude or spindle rhythm activities and consists of low-frequency delta $(0-4 \mathrm{~Hz})$ activities. REM sleep is defined by high rhythmic activity at theta band $(4-8 \mathrm{~Hz})$ and relatively low EMG activity. A few EMG twitches can be found during REM sleep, which was calibrated by video recordings.

\section{Visualized Movement Detection}

As neck EMG does not fully capture motor activity of animal, eg limbs movements, a Python routine was developed to quantitatively analyze body movements in video recordings that synchronized with EEG and EMG recordings. To calibrate the analysis accuracy, a video acquisition system was settled on every cage to monitor rats behavior. Videos were captured in $512 \times 512$ frame size and with a recording speed of 50 frames per second. The number of pixels that changed in greyscale between two video frames was counted. The mean number of pixels involved in REM sleep motor events in each REM sleep episodes was calculated during recording.

\section{REM Sleep Deprivation and Rebound}

To study physiological conditions during REM sleep, rats were undergoing 48 hours REM sleep deprivation, using the widely applied platform method. ${ }^{37}$ Rats that underwent REM sleep deprivation were placed on a small platform with $7 \mathrm{~cm}$ in diameter, which is surrounded by a pool of water $\left(37^{\circ} \mathrm{C} ; 2 \mathrm{~cm}\right.$ high). Muscle paralysis during REM sleep could result in the consequence of fall into water and successfully depriving most of REM sleep. Control animals have a larger platform of $15 \mathrm{~cm}$ diameter, which allows for sleep.
Microinjection of Adeno-Associated Virus (AAV)

The viral vector AAV8-CaMKIIa-hM3Dq-mCherry and AAV8-CaMKIIa-mCherry were obtained from the Vector Core of the University of North Carolina (Chapel Hill, NC, USA). Rats were anaesthetized with $4 \%$ chloral hydrate (40 $\mathrm{mg} / \mathrm{kg}$ ) and secured in a stereotaxic apparatus. Microinjection of adeno-associated viruses (AAV) was performed using a 27-gauge Hamilton micro-syringe. The injection was firstly targeted at bilateral SLD; coordinates and injection volume were antero-posterior (AP) $-8.80 \mathrm{~mm}$; medio-lateral (ML) $\pm 1.25 \mathrm{~mm}$; dorso-ventral (DV) $7.00 \mathrm{~mm}$ and $0.5 \mu \mathrm{L}$ per injection. Microinjection targeting other brain regions will refer to coordinates listed in Stereotaxic Coordinates (Rats). After AAV microinjection, EEG and EMG electrodes were implanted as described in Method d. After microinjection and implantation, the skin of the surgery regions was restored by dental cement. Together with pain relief, 80k unit penicillin was applied subcutaneously twice daily for 7 days. All rats had a period of 14-days convalescence after surgery for gene expression before data acquisition began.

\section{Designer Receptors Exclusively Activated by Designer Drugs (DREADD)}

To activate GPCR labelled neurons, we injected CNO dissolved in $0.1 \mathrm{~mL}$ vehicle solution into rats expressing hM3Dq in the SLD, 30 min before the recording session. According to changes in $\mathrm{hM} 3 \mathrm{Dq}$ transfected rats' sleep structure when applying different dosages of CNO. In EEG recording, we found an increased REM sleep proportion to total sleep in $\geq 2 \mathrm{mg} / \mathrm{kg}$ dosage of CNO administration, which lasted for $\sim 2$ hours. $\mathrm{CNO}$ was administered intraperitoneally at $2 \mathrm{mg} / \mathrm{kg}$ dose on different days of modeling. Two control groups were settled for any undesired effects of hM3Dq transfection and CNO injection, respectively. One control group received $\mathrm{AAV}-\mathrm{hM} 3 \mathrm{Dq}$ and $\mathrm{CNO}$ solvent (ie $1 \%$ DMSO in de-ionized water) to control undesirable effects of hM3Dq transgene. Another control group received AAV-mCherry and $2 \mathrm{mg} / \mathrm{kg} \mathrm{CNO}$ to control undesirable effects of CNO. Animals were subjected to the same conditions in all recordings. EEG \& EMG recording will start 0.5 hours after $\mathrm{CNO}$ injection and lasted for 6 hours during the light phase. To minimize any acute effect of rotenone, rats received daily rotenone injection after the 6-hour EEG/EMG recording session. Rats were entered the next circadian period after all the above-mentioned steps. 


\section{Immunostaining}

At the endpoint of modeling and in vivo experiments, animals were perfused under deep anesthesia with phosphate buffer (PB), followed by a fixative $400 \mathrm{~mL}$ of $4 \%$ paraformaldehyde solution in $0.1 \mathrm{M} \mathrm{PB}(\mathrm{pH}$ 7.4). The brains were mounted by cryo-embedding media and frozen with liquid nitrogen, then stored at $-80^{\circ} \mathrm{C}$. Coronal sections $(30 \mu \mathrm{m})$ were obtained with a freezing microtome. All the free-floating sections were permeabilized with PBS containing $0.1 \%$ Triton X100 for $15 \mathrm{~min}$, then washed with PBS and blocked by $2 \%$ normal goat serum (NGS) for $1 \mathrm{~h}$ at room temperature. After blocking it, all floating sections were washed with PBS for three times and then incubated with the primary antibody in $2 \% \mathrm{NGS}$ at room temperature for $30 \mathrm{~min}$ and placed at $4{ }^{\circ} \mathrm{C}$ overnight. Concentrations of primary antibodies were diluted to: 1:500 for anti-vGluT2, 1:500 for anti-GAD67, 1:500 for anti-GlyT2, 1:1000 for anti-alpha synuclein and 1:500 for c-fos. After primary antibody incubation, sections were washed by PBS for three times and then incubated with corresponding secondary fluorescence antibodies for single or double labelling at room temperature for 2 hours. After secondary incubation, sections were washed and incubated with 1:10,000 4',6-diamidino-2-phenylindole (DAPI) for $30 \mathrm{~min}$. After the above steps, all sections were mounted onto super-frost slides and then labeled and cover-slipped for confocal imaging.

\section{TUNEL Staining}

TUNEL labeling was performed with an ApopTag1 detecting kit and followed manufacturer's instructions (Millipore, USA). ${ }^{38}$ The brains were mounted with paraffin and sectioned to $7 \mu \mathrm{m}$ thick coronal slices containing dorsal pontine and ventral medullary regions. TUNEL staining was performed on one section of five consecutive sections. After the staining procedure, the slides were mounted with Permount medium and observed under a light microscope (Zeiss Microscope Axiophot 2).

\section{In-Situ Hybridization (ISH) for vGluT2 mRNA Combined with Immunostaining}

The fluorescence-labeled probe against vGLUT2 mRNA slc17a6 were purchased from LGC Biosearch (CA, USA). As described in method (e), brain sections were successively prepared and immersed for around $10 \mathrm{~min}$ in PBST containing $0.05 \%$ ribonucleoside vanadyl complex (VRC, Sigma-Aldrich, St. Louis, MO) and $0.02 \%$ Triton. They were then rinsed in PBST and in standard saline citrate solution (SSC 2X), respectively. Sections were then placed at $37^{\circ} \mathrm{C}$ for around 12 hours in the hybridization buffer (SSC $2 \mathrm{X}$ with $50 \%$ formamide and $0.1 \%$ Tween-20) containing $0.5 \mu \mathrm{g} / \mathrm{mL}$ of the fluorescence-labeled probe. Sections were washed in SSC 2X, 50\% formamide, $0.1 \%$ Tween- 20 and $\mathrm{SSC} 1 \mathrm{X}$ at $37^{\circ} \mathrm{C}$, then rinsed by SSC $4 \mathrm{X}$ in room temperature. Sections were then moved to PBST and proceed with immunofluorescence procedures in method (e). ISH labelled sections incubated with a mouse antiserum for alpha synuclein (1:2000; Merck, Germany), a biotinylated goat anti-mouse $\operatorname{IgG}$ solution labelled by AlexaFlour 488 dye (Thermo Fisher, USA). Controls in the absence of anti-alpha synuclein antibody or with the sense probe were run to ensure the specificity of the labeling.

\section{Statistical Analysis}

Statistical analysis was performed using SPSS (version 10.0, SPSS Inc. Chicago, IL) and GraphPad Prism (GraphPad Software, LLC). All progressive Parkinsonism modeling results are presented as mean $\pm \mathrm{SEM}$, and then determined by one-way ANOVA followed by Tukey's test for multiple comparisons (Figures 1-5). All chemogenetic results are compared with day 0 or sham groups and determined by two-way ANOVA followed by Tukey's test for multiple comparisons (Figures 6 and 7). In progressive Parkinsonian modeling, the $\mathrm{p}$ value was determined from the deviation of the observed value vs day 0 . In chemogenetic studies, the $\mathrm{p}$ value was determined from the deviation of the observed value vs day 0 or sham groups.

\section{Results}

\section{Increased Motor Behavior During Sleep in the Rotenone Model of Parkinson's \\ Disease}

Rats that underwent daily intraperitoneal injection of $2.5 \mathrm{mg} / \mathrm{kg}$ rotenone exhibited signs of motor deficits progressively. After two weeks of rotenone treatment, there were a significantly reduced number of movement episodes in the open field (Supplementary Figure 1A) and reduced latency to fall from the rotarod from $78.3 \pm 8.8 \mathrm{~s}$ to $17.9 \pm 5.0 \mathrm{~s}$ (Supplementary Figure 1B). The performance further deteriorated at the end of 4 weeks of treatment. Tyrosine hydroxylase expression was significantly decreased in the substantia nigra para compacta after the treatment period (Supplementary Figure 1C). 
At the same time, based on EMG recordings and video monitoring, we found evidence of increased motor behavior during sleep. Vigilance states were analyzed in each 10-seconds sleep episodes, and identified by wakefulness, REM sleep and NREM sleep (Supplementary Figure 2). In REM sleep, the muscle atonia (or non-active) is defined as the inhibition of muscle tone in 10-second sleep epochs compared to the NREM sleep. "Active" sleep episodes are defined by a 10-second REM or NREM episode that contains muscle twitches that $15 \%$ higher than the averaged REM sleep EMG integral in sham rats and visualized movements exampled in Video 2. Only REM sleep that sustained longer than $30 \mathrm{~s}$ was considered to prevent contamination from wakefulness. As demonstrated by typical EMG recordings of the neck muscle (Figure 1A left), all REM sleep episodes were divided as containing motor behavior (active) or not (non-active). The percentage of active REM sleep episodes was significantly increased after two weeks of rotenone treatment from $7.8 \pm 2.1 \%$ to $24.0 \pm 2.2 \%$ (Figure 1B).

In this model, NREM muscle twitches were also observed. After 4 weeks of rotenone treatment, a few consecutive and frequent muscle activities were recorded in some NREM sleep episodes (Figure 1A). However, increased muscle activity was more prominent in REM sleep, as indicated by the increased EMG integral from $1.06 \pm 0.11$ to $1.46 \pm 0.15$ during REM sleep over NREM sleep EMG integral after 2 weeks of rotenone administration (Figure 1B). Apart from increased neck muscle activity during REM sleep, increased gross body movement was also found, which was gauged by the mean number of changed grey-scale pixels in every second of paradoxical sleep in the video monitoring (Figure 1C). In regular REM sleep without sleep behaviors, changed greyscale pixels were $546.7 \pm 125.4$ per $10 \mathrm{~s}$ (Supplementary Video 1). Pixels involved in REM sleep motor behaviors were increased to $3761.3 \pm 950.9$ per $10 \mathrm{~s}$ (Supplementary Video 2).

\section{Altered Sleep Architecture in Rotenone-Induced Parkinsonism}

In addition to the appearance of REM sleep behavior, we also detected changes in the sleep-wake cycle and sleep architecture in our chronic PD model. After 4 weeks of rotenone treatment, rats spent more time in wakefulness (ie less sleep) during the daytime that corresponds to their inactive/sleeping phase, especially before entering the night-time while there was not much difference during the night-time, or active phase (Figure 2A-C). During the daytime, both REM and NREM were significantly reduced. A compensatory increase in NREM sleep but not REM sleep was exhibited in subsequent night-time recordings. Moreover, since the duration of NREM and REM episodes decreased gradually towards the late stages of rotenone treatment (Figure 2D and E), increased number of arousals beginning at the end of second week were observed (Figure 2F), which indicated fragmentation of sleep.
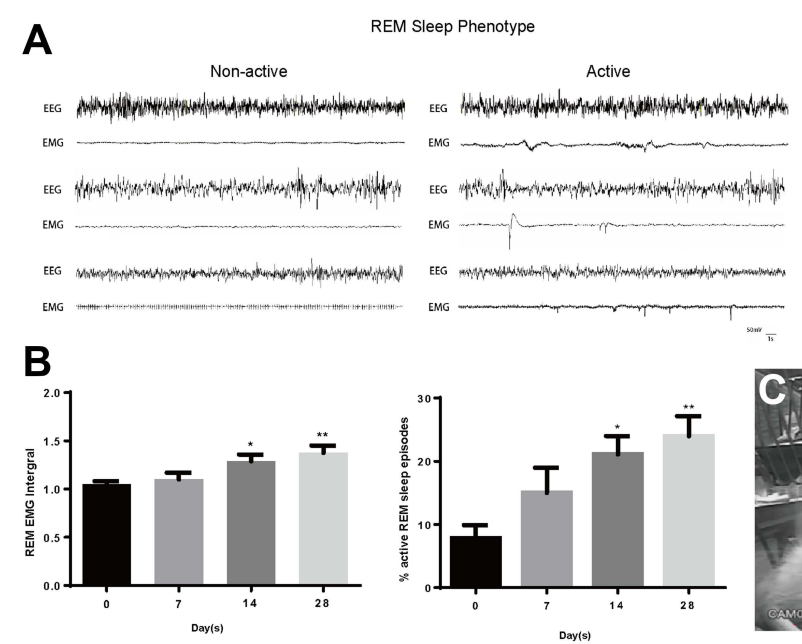

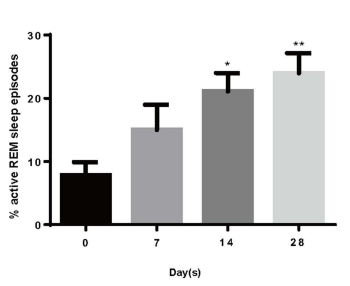

NREM Sleep Phenotype
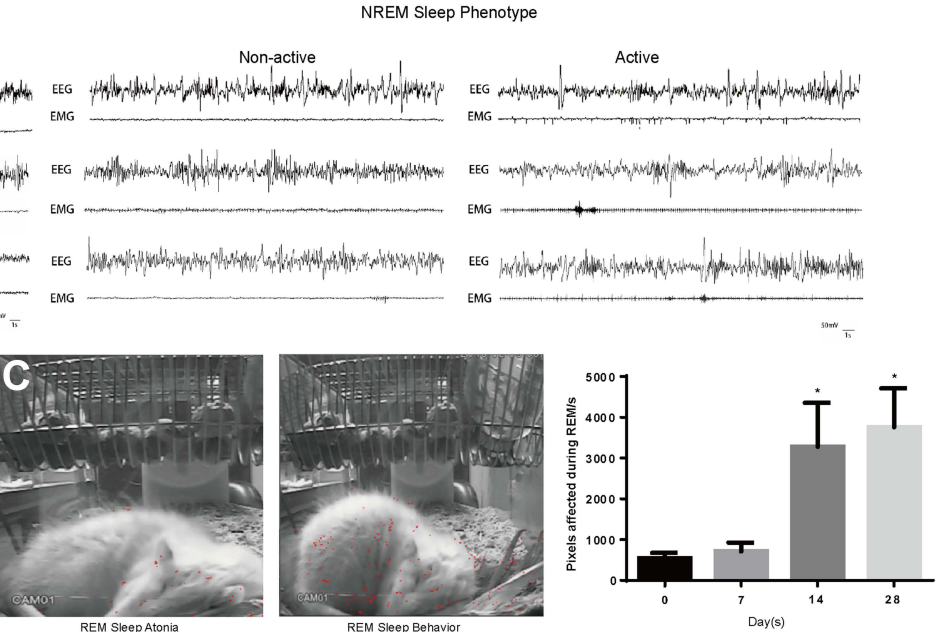

Figure I Increases of sleep behaviors with developing PD symptoms. (A) Typical traces of EEG and EMG recordings showing that motor behaviors occurred in REM sleep (left panel) and NREM sleep (right panel). (B) REM sleep episodes containing REM sleep behaviors increased after I4 days of rotenone treatment, as indicated by the increase in REM EMG integral and increased percentage of active REM episodes. (C) In analysis of movement by video recording, REM sleep behavior was indicated by increased numbers of changed pixels, which are marked in red. $n=6,{ }^{*} P<0.05, * * P<0.01$, one-way ANOVA with Tukey's test. 
A

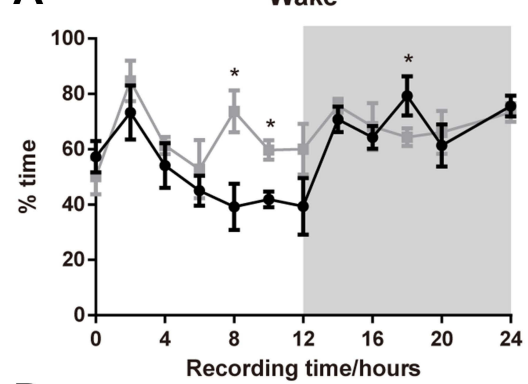

D
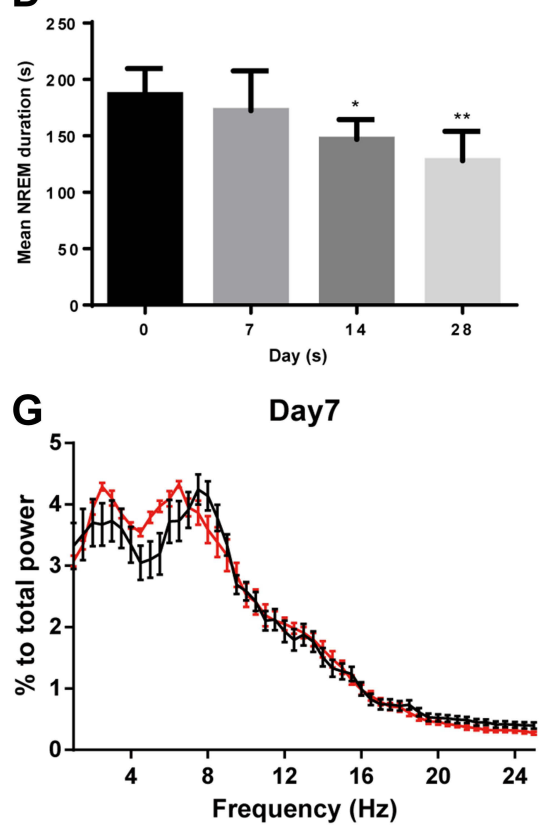

B

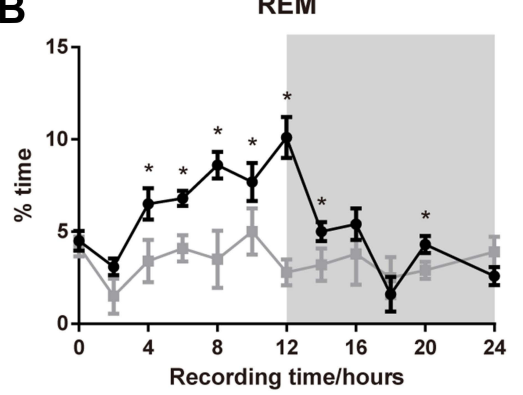

E

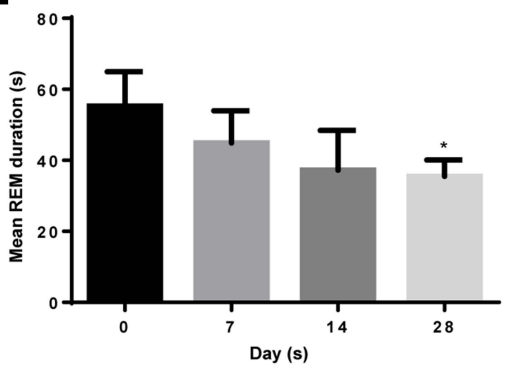

Day14

H

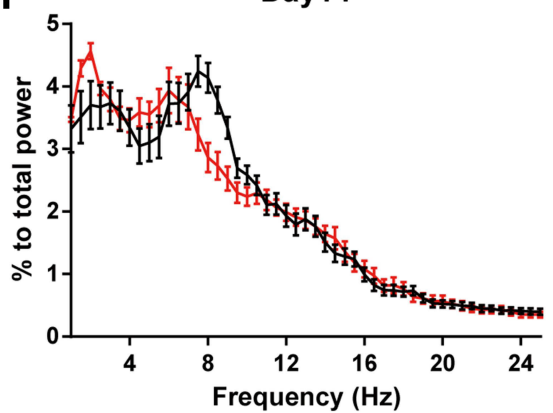

C

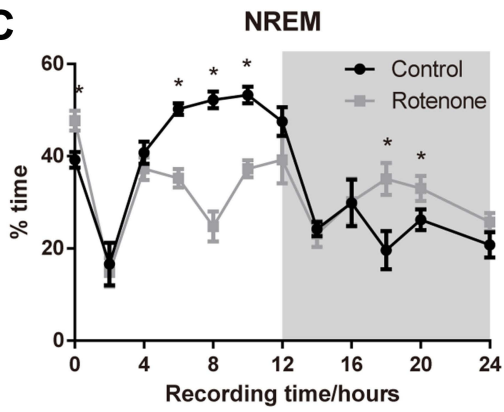

$\mathbf{F}$

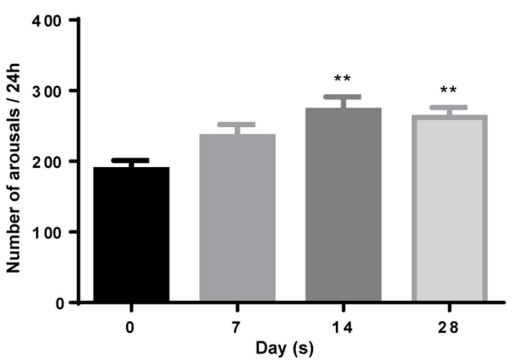

Day28

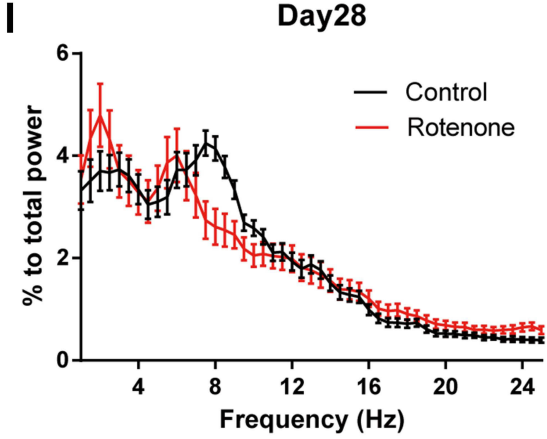

Figure 2 Changes in sleep architecture in chronic rotenone PD model. (A-C) Rats receiving 28 days of rotenone treatment exhibited disturbed circadian rhythm in 24 hours recording. (D and E) The mean durations of both NREM and REM sleep episodes decreased during the modeling period. (F) Number of arousals was also increased after 2 weeks of treatment. $(\mathbf{G}-\mathbf{I})$ The power spectrum exhibited slowed peak of theta rhythm $(4-8 \mathrm{~Hz})$ appearing after 7 days and tested the whole modeling period. $\mathrm{n}=$ 6 per group, ${ }^{* P}<0.05,{ }^{*} * \mathrm{P}<0.01$, Two-way ANOVA for $(\mathbf{A}-\mathbf{C})$, one-way ANOVA with Tukey's test for (D-F).

RBD patients always have cortical EEG shifted to lower frequencies, implicating association between $\mathrm{RBD}$ and neurodegenerative diseases. ${ }^{39,40}$ We therefore also analyzed and compared the spectral properties of the EEG signals between control and rotenone-treated groups. In the fronto-parietal region, the peak frequency of theta EEG $(4-8 \mathrm{~Hz})$ in control group was $\sim 7.5 \mathrm{~Hz}$ while in the rotenone treated rats, the theta peak frequency was gradually left-shifted to $\sim 5.5 \mathrm{~Hz}$ as the rotenone treatment and Parkinsonian symptoms progressed. The spectral densities of EEG signals in the first, second and fourth weeks compared with the day 0 animals are shown in Figure 2G-I. Comparison of RBD symptoms in rotenone model and RBD patients are shown in Table 1.

\section{Accumulation of Alpha-Synuclein and Apoptosis in REM Sleep Regulatory Nuclei}

Our results suggest that REM sleep regulatory circuitry may be damaged by chronic rotenone treatment. We thus examined whether alpha-synuclein, which is regarded as toxic, was accumulated in relevant brain nuclei. The target areas were the pontine REM-on nucleus SLD and its downstream target gigantocellular ventricular reticular nucleus (GiV) in the medulla. Immunostaining revealed that alpha-synuclein clearly accumulated in the SLD glutamatergic neurons, which were identified by co-immunolabelling for vGluT2 (Figure 3A). A significant increase in the alpha-synuclein fluorescence signal was found two weeks after rotenone treatment and increased further after 4 weeks by $173 \%$ 
Table I Chronic Rotenone Modeling in Rats Exhibited Key Symptoms of RBD Analogous to Key RBD Features in Clinical Observation

\begin{tabular}{|l|l|l|}
\hline Human Symptoms & Symptoms Observed in Rats & Identifying Parameter in Rats \\
\hline $\begin{array}{l}\text { Dream enactments, often violence in motor } \\
\text { behaviors }\end{array}$ & $\begin{array}{l}\text { Increased active REM sleep } \\
\text { episodes }\end{array}$ & $\begin{array}{l}\text { Elevated muscle tones in REM sleep and visualized } \\
\text { behaviors }\end{array}$ \\
\hline Increased NREM sleep movements & $\begin{array}{l}\text { Increased NREM sleep muscle } \\
\text { tones }\end{array}$ & Elevated muscle tones in NREM sleep \\
\hline Sleep fragmentation & Sleep fragmentation & Increased number of sleep episodes in 24 hours \\
\hline EEG slowing & EEG slowing & Reduced Theta-band frequency \\
\hline
\end{tabular}

(Figure 3B). In the GiV, which is mainly populated by GABAergic neurons and identified by GAD67 immunostaining (Figure 3C), we did not find an increase in alpha-synuclein expression level after 2 weeks of rotenone treatment. However, alpha-synuclein started to accumulate after 4 weeks of treatment and increased by $141 \%$ in fluorescence intensity (Figure 3C and D). These results indicate abnormal accumulation of alpha-synuclein in the key REM sleep regulatory centre SLD and also its downstream target GiV, which has been implicated in controlling REM sleep atonia.

At the same time, we also monitored the degree of neuronal apoptosis in SLD and GiV, and we conducted TUNEL staining on these two specific nuclei 2 and 4 weeks after rotenone treatment. We found a significantly increased number of apoptotic neurons in the SLD from the second week and further increased in 4th week (Figure 4A and B). Compared with day 0 , the percentage of observed TUNEL positive cells after 2 weeks increased from $4.57 \pm 1.46$ to $24.86 \pm 4.14$, and then increased to 38.14 \pm 6.15 after 4 weeks. In contrast, in the GiV, a significant increase in the number of neurons undergoing apoptosis was found only after 4 weeks of rotenone treatment from 3.29 \pm 1.23 to $34.86 \pm 3.84$ (Figure $4 \mathrm{~B}$ ). The profiles of apoptosis in these two areas are therefore highly parallel to those of alpha-synuclein accumulation, which together suggest that SLD is affected by rotenone at an earlier time than GiV.

\section{Alterations in Neural Activities of Sublaterodorsal Nucleus and Gigantocellular Ventricular Reticular Nucleus}

The relatively early accumulation of alpha-synuclein, increase in apoptosis and also decrease in EEG frequency strongly implicate that rotenone administration has a major impact on the integrity of the REM-on nucleus SLD. To probe whether the function of this nucleus is compromised under rotenone treatment, we examined the degree of neuronal activation by c-fos expression in sleep deprivation induced REM sleep
A

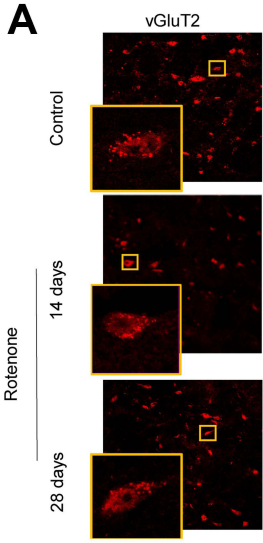

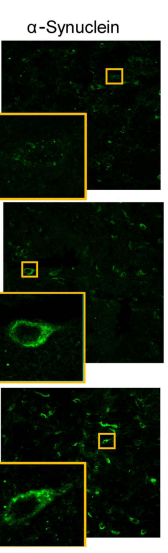
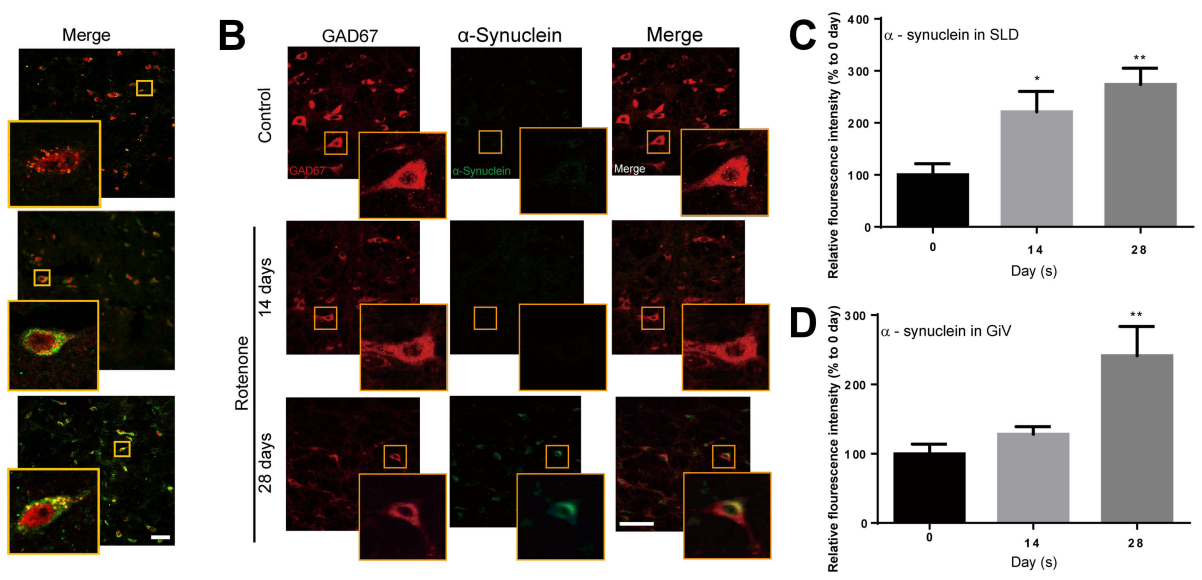

Figure 3 Progressive alpha-synuclein aggregation in SLD glutamatergic neurons and GiV GABAergic neurons. (A and B) (A) Cytoplasmic inclusion of alpha-synuclein in SLD glutamatergic cells appeared after 2 weeks of rotenone treatment while (B) alpha-synuclein aggregation in GiV GABAergic cells appeared after 4 weeks. (C and D) Quantification of cytoplasmic fluorescence intensity of alpha-synuclein in (C) SLD and (D) GiV; $\mathrm{n}=6$ per group, *P<0.05, **P<0.0I, compared with Day 0, one-way ANOVA with Tukey's test. 

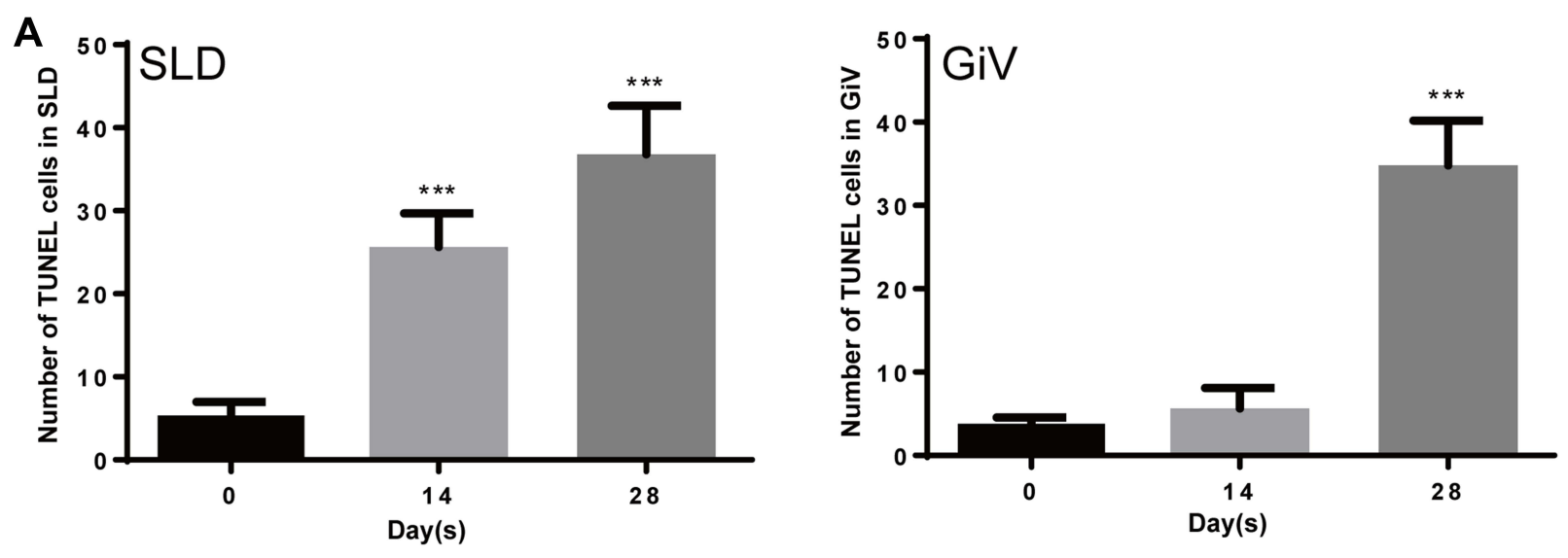

B
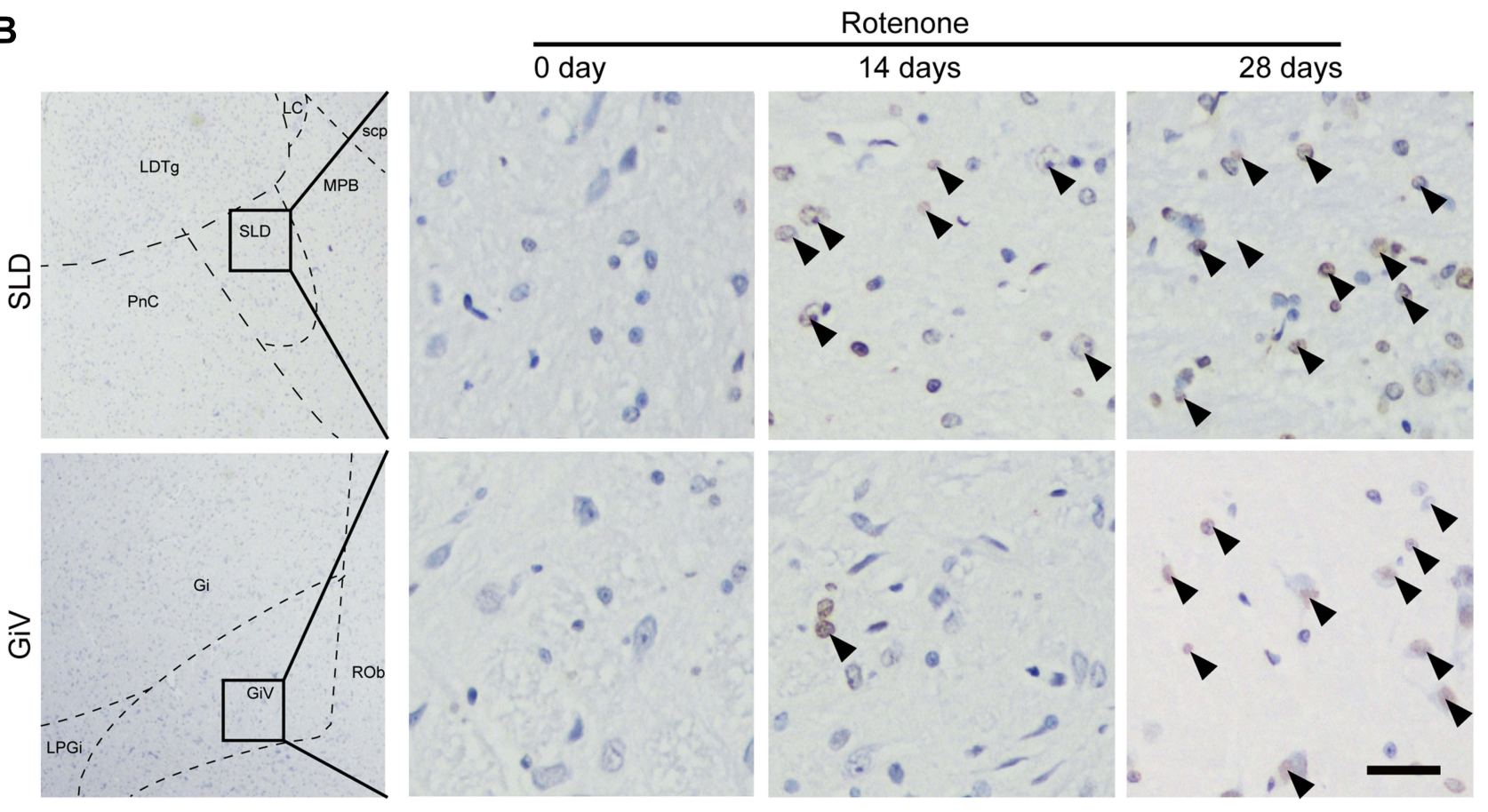

Figure 4 Neuronal apoptosis in REM sleep atonia pathway during rotenone treatment. (A) Number of TUNEL+ cells in SLD increased after 2 weeks and in GiV after 4 weeks. (B) Examples showing increases of TUNEL+ cells in SLD and GiV (arrows). $n=6$ per group, ***P<0.00I, one-way ANOVA with Tukey's test. Scale bar: I00 $\mu$ m.

hypersomnia, a period during which SLD is highly activated.

We found that REM sleep hypersomnia emerged during rebound period of REM sleep deprivation. To identify the glutamatergic nature of the neurons examined, we co-stained the neurons for vGlut2 expression. As expected, REM sleep deprivation increased REM sleep amount to $27.6 \pm 3.9$ (Figure $5 \mathrm{~A}$ ) in the subsequent 2 hrs. The animals were sacrificed at the end of the second hr. Before treatment, large numbers of c-fos + labeled cells were found in SLD. In contrast, in the rats treated with rotenone for 2 weeks, the amount of c-fos + labeled cells was reduced from $26.1 \pm 5.3$ to 18.4 \pm 2.1 ; after 4 weeks, c-fos + labeled cells in SLD was reduced to $15.7 \pm 3.4$ (Figure $5 \mathrm{~B}$ and $\mathrm{C}$ ).

\section{Activation of SLD Neurons Ameliorates RBD Symptoms in Rotenone PD Model}

To confirm the causal relationship between PD-affected SLD neuronal activity and RBD symptoms, chemogenetic manipulation was conducted. In another group of rats, AAVCaMKIIa-hM3Dq-mCherry was micro-injected into SLD (Figure 6A and B). Bilateral SLD glutamatergic neurons were activated by injection of clozapine-N-oxide (CNO) during the light phase. Figure 6C summarizes the schedule of chemogenetic activation of SLD neurons. Rotenone was 
A

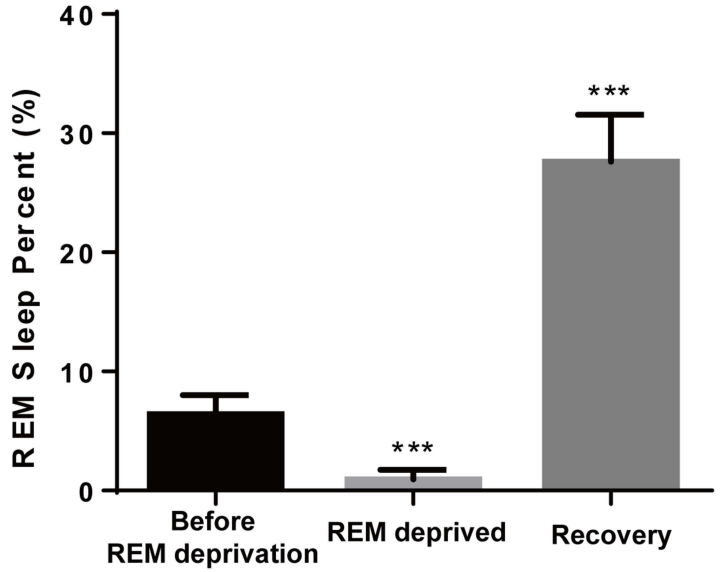

B

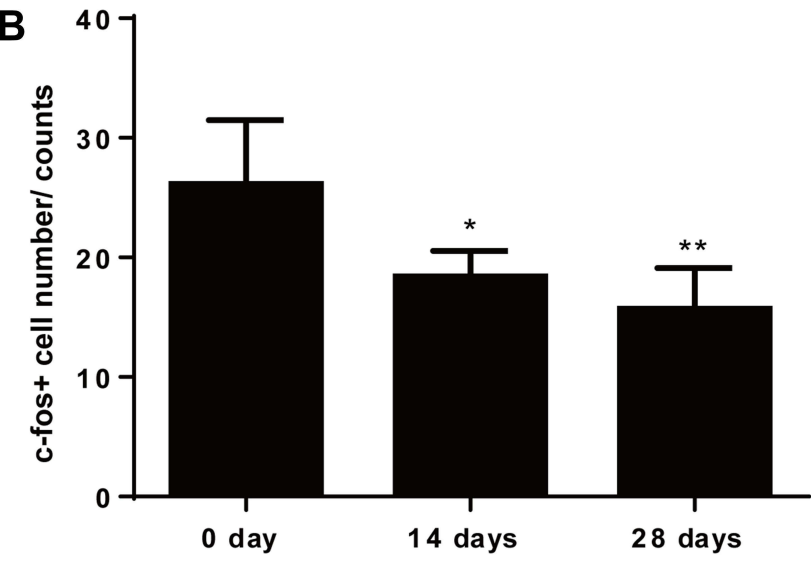

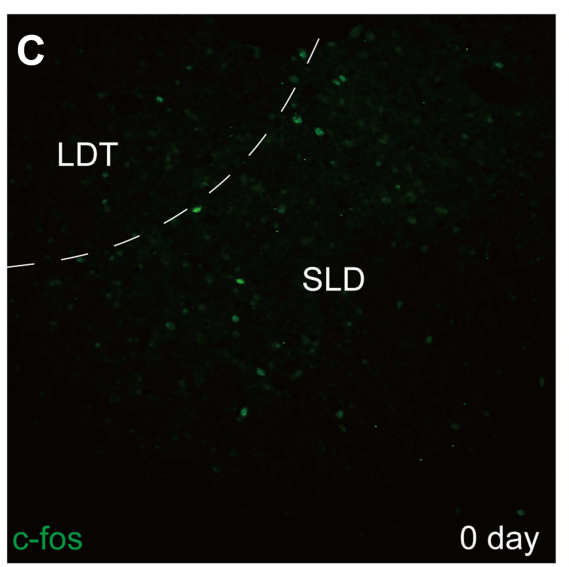
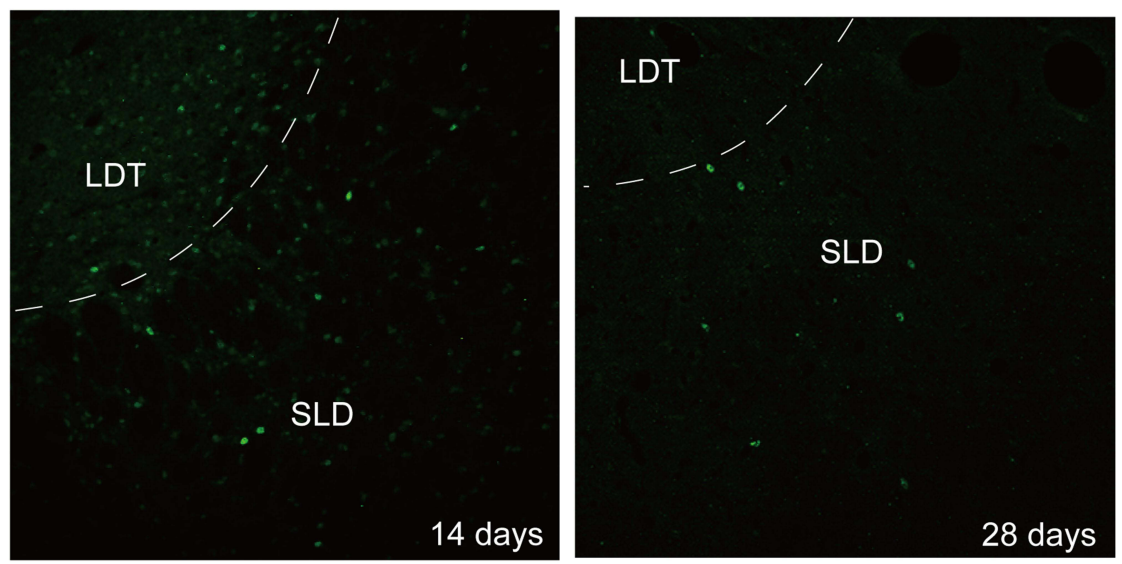

Figure 5 Decreased c-fos expression in SLD during REM sleep rebound. (A) REM sleep increased when recovering from REM sleep deprivation. (B) c-fos expression in SLD decreased progressively from 14 days to 28 days in the PD model. (C) Examples showing c-fos expression in SLD during REM sleep rebound, in different groups. $\mathrm{n}=6$ per group, ${ }^{*} \mathrm{P}<0.05, * * \mathrm{P}<0.01, * * * \mathrm{P}<0.001$, one-way ANOVA with Tukey's test. Scale bar: $100 \mu \mathrm{m}$.

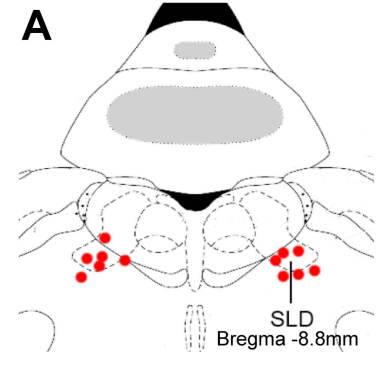

C

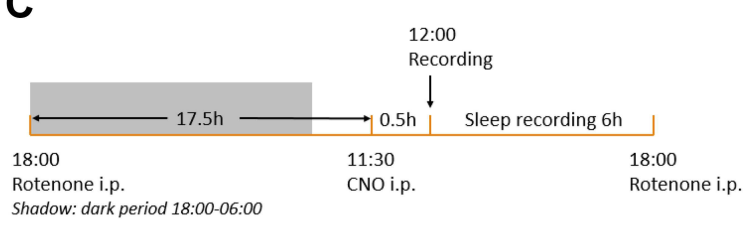

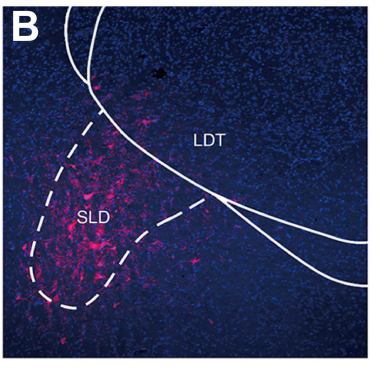
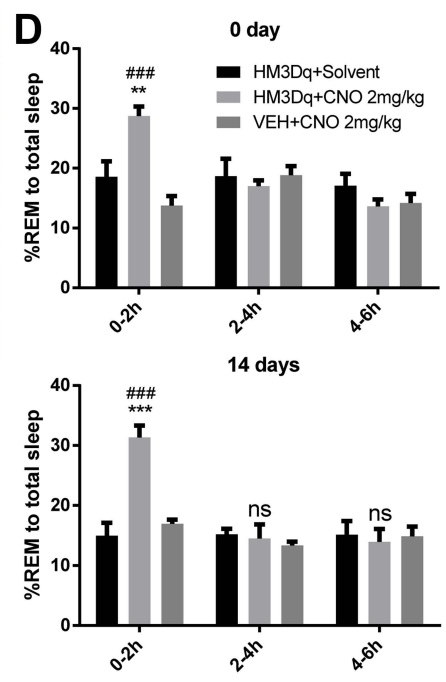

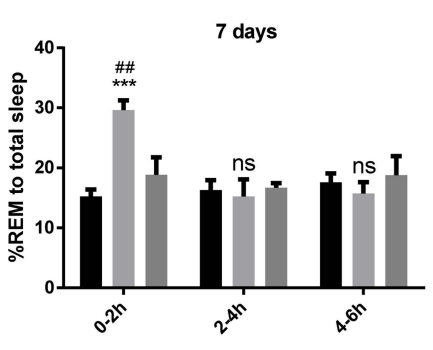

28 days

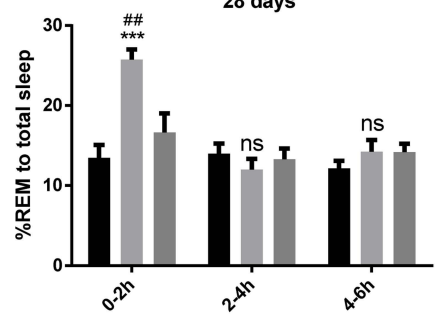

Figure 6 Effect of chemogenetic activation of SLD glutamatergic cells. (A) Injection site of AAV carrying hM3Dq targeting the SLD area. (B) hM3Dq-mCherry expressed in the SLD. (C) The schedule of chemogenetic activation of SLD neurons (D) Proportion of REM sleep in the total sleep increased at the first 2 hours after CNO injection, in different stages of rotenone treatment. n = 6 per group, ns, not significant, ${ }^{\#, ~ C o m p a r e ~ t o ~ V E H+C N O ~ g r o u p, ~} * * P<0.0$ I, ${ }^{* * *} \mathrm{P}<0.00 \mathrm{I}$, one-way ANOVA with Tukey's test. 
injected after daily EEG \& EMG recordings. When compared with the two control groups that received CNO solvent and transfected with mCherry only, the proportion of REM sleep in total sleep was drastically increased from $18.6 \pm 2.6 \%$ to $28.7 \pm 1.6 \%$ during first 2 hours of $\mathrm{CNO}$ injection and the increase in REM sleep proportion lasted in the entire PD modeling session of 4 weeks (Figure 6D).

Next, we evaluated the proportion of EMG-active REM sleep episodes during SLD glutamatergic neuron activation. REM sleep behaviors in rats with CNO administration were compared with two control groups. As discussed above, chronic rotenone modeling triggered RBD symptoms in 2 weeks, which paralleled the appearance of active REM episodes after 2 weeks of rotenone exposure, which were also found in vehicle $+\mathrm{CNO}$ control group (Figure 7A). With chemogenetic activation of SLD, sleep episodes containing REM behaviors were suppressed (Figure 7B). However, an increase in active REM sleep episodes and REM EMG integral still occurred but was postponed to after 4 weeks of rotenone treatment (Figure 7C). These results suggest that activation of SLD glutamatergic neurons could alleviate RBD symptoms in developing Parkinsonism. Also, the reduced REM sleep theta frequency caused by 4 weeks of rotenone exposure was partly restored from $5.33 \pm 0.33 \mathrm{~Hz}$ to $6.92 \pm 0.27 \mathrm{~Hz}$ by the activation of SLD glutamatergic neurons (Figure 7D).

\section{Discussion}

In this study, we found that the chronic rotenone model of PD recapitulates some prominent features of RBD. The correlation between the time course of deposition of alpha-synuclein and RBD symptoms is consistent with a progressive degeneration in the brainstem REM sleep promotion and the atonia circuit in early Parkinsonism that underlies the emergence of RBD symptoms, strengthening the proposition that RBD could serve as a biomarker for PD. Therefore, the rotenone model could serve as a useful experimental model for investigating the mechanism of RBD and also its relationship to neurodegenerative diseases.

RBD patients often suffer from various sleep disruptions, including sleep fragmentation, losses in REM sleep and excessive daytime sleepiness. ${ }^{1,41}$ Here, the major sleep disturbances associated with RBD, including sleep fragmentation and losses of diurnal variation, were observed at
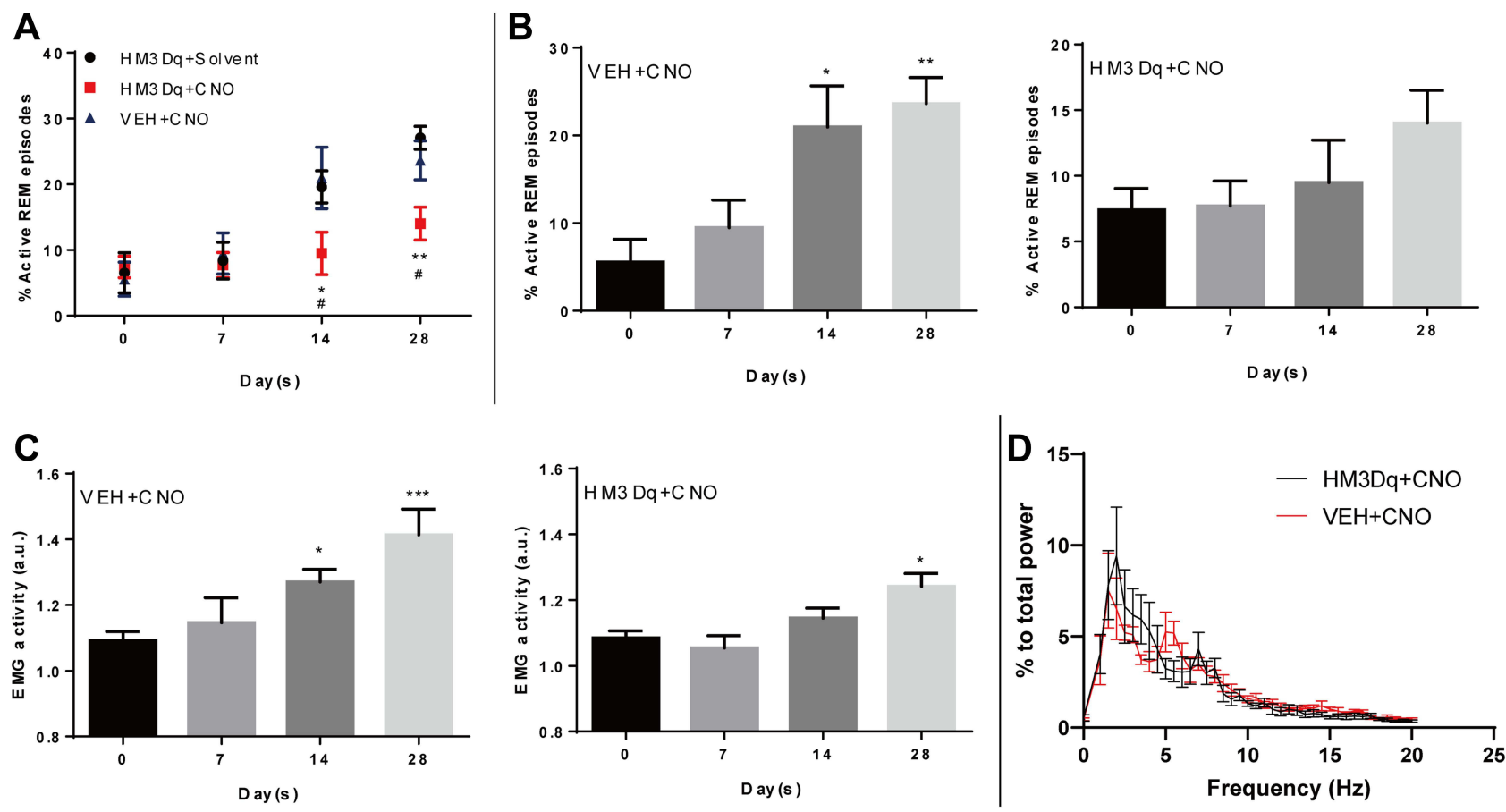

Figure 7 Activation of SLD glutamatergic neurons postponed and alleviated RBD symptoms. (A) Active REM sleep episodes decreased after 2-weeks of rotenone modeling when compared with vector or $\mathrm{CNO}$ control groups. Two-way ANOVA with Tukey's test, ${ }^{*}$ Comparison between $\mathrm{HM} 3 \mathrm{Dq}+\mathrm{CNO}$ and $\mathrm{VEH}+\mathrm{CNO}$; ${ }^{\mathrm{p}}<0.05$; **p $<0.0 \mathrm{I}$; $\mathrm{n}=6$ in each group. (B) Chemogenetic activation of SLD glutamatergic neuron showed no increases of active REM episodes throughout the modeling periods, in contrast, CNO control group showed increases of active REM episodes from 14 days of rotenone exposure. One-way ANOVA with Tukey's test, *p<0.05; **p< 0.01 . (C) Chemogenetic activation of SLD glutamatergic neuron postponed REM EMG integral increases to 4 weeks after rotenone exposure. One-way ANOVA with Tukey's test, * $<0.05$, *** $\mathrm{p}<0.00 \mathrm{I}, \mathrm{n}=6$ in each group. (D) Comparison of REM sleep power spectral on 4 weeks of rotenone exposure, theta slowing was clearly alleviated comparing with rotenone treated group. $n=6$ in each group. 
different time points of the chronic PD model induced by rotenone. Sleep fragmentation and losses in diurnal variation indicate deficits in wake-on and sleep-on regions, which impairs REM-NREM antagonism. ${ }^{42,43}$ On the other hand, the reduction in REM sleep suggests lesions in REM sleeppromoting areas. ${ }^{44}$ Reduced frequencies in power spectral rhythms were observed in both RBD and PD patients, ${ }^{40}$ which suggest reduced ascending projections from pons. ${ }^{45,46}$ Previous imaging studies suggested reduced brain blood flow in RBD patients with a reduction in waking EEG activities. ${ }^{47}$ There are hypotheses that EEG slowing is triggered by changing inhibitory projections to cortical regions. ${ }^{14,48}$ In the rotenone-treated rats, power spectral analysis also revealed theta rhythm slowing and increased beta rhythm during REM sleep. In this model, reduction of Theta frequency occurred after just 1 week of treatment and prior to all other RBD or PD symptoms. We therefore propose that early EEG slowing might signify changes in subcortical-tocortical projections and could serve as a sensitive indicator for RBD.

It has been well documented that chronic application of rotenone induced alpha-synuclein aggregation in substantia nigra, recapitulating motor Parkinsonian motor symptoms. $^{33,49}$ However, accumulated alpha-synuclein may affect not only catecholaminergic neurons but also glutamatergic neurons and synapses. ${ }^{50,51}$ In this study, we presented evidence that REM sleep circuit is affected by alpha-synuclein aggregation. First, after 14 days of rotenone treatment, alpha-synuclein aggregation and apoptosis are found in SLD glutamatergic neurons, which may severely impair its output to other areas, leading to disrupted sleep architectures and impairment of REM sleep atonia. ${ }^{19}$ Second, we demonstrated cytoplasmic inclusion of alpha-synuclein and also apoptosis in GABAergic neurons in GiV and reduced expression of GAD67, respectively, suggesting impaired functions of these neurons that are known to project to the spinal cord and cause atonia. In fact, losses of medullary GABA neurons had been reported in PD patients. ${ }^{52}$

The above findings are consistent with reduced c-fos expression in SLD and GiV during REM sleep rebound following sleep deprivation. It is known that during REM sleep, SLD glutamatergic neurons and GiV GABAergic/ glycinergic neurons burst to maintain REM sleep atonia and NREM/REM antagonism. ${ }^{36,53}$ At the same time, we showed that activation of SLD glutamatergic neurons could effectively suppress or postpone RBD symptoms in the animals. These results consolidate previous findings that glutamatergic projection from SLD innervate downstream muscle atonia circuitry ${ }^{17,54,55}$ and that REM sleep behavior may be triggered by loss-of-functions of SLD in early developing neurodegeneration. ${ }^{11}$

In this study, we showed that PD development triggers progressive pontine-medullary dysfunction and disrupts the

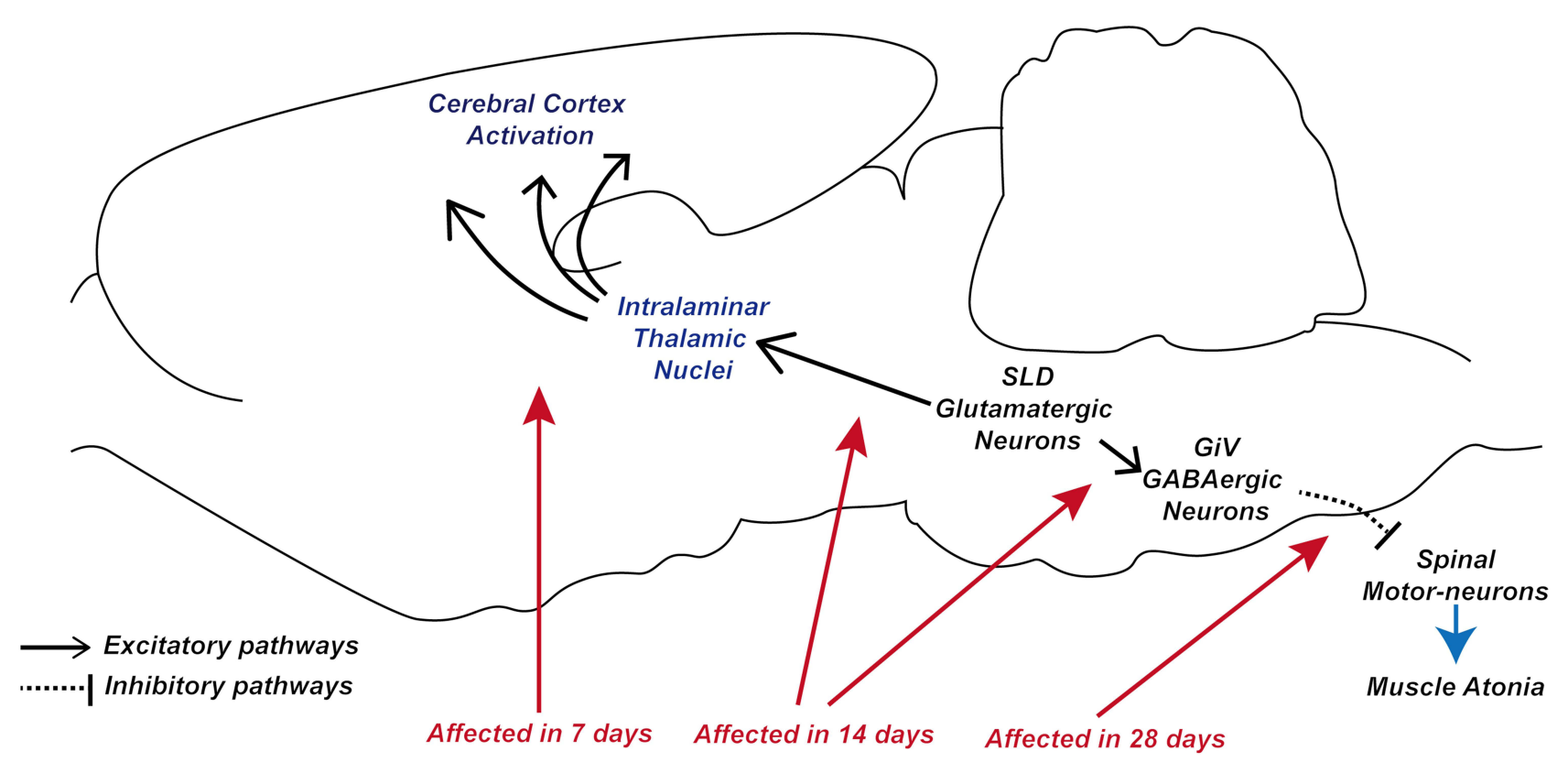

Figure 8 A putative mechanism of RBD in chronic rat PD model. After 7 days of rotenone treatment, no increase of REM sleep behavior observed but only mild theta-wave slowing during REM sleep. After 14 days of treatment, key RBD features including REM sleep behavior and sleep fragmentation exhibited due to damage of SLD glutamatergic neurons. After 28 days, RBD symptoms worsens with appearance of abnormal NREM muscle twitches, with impairment in medulla inhibitory projections. 
maintenance of REM sleep muscle atonia, but our results are limited to certain critical brain structures and not the whole circuitry. $^{16}$ SLD activity is modulated by inhibitory GABAergic inputs from the laterodorsal tegmental nucleus (LDT) and the ventrolateral periaqueduct grey (vlPAG), which forms an on/off switch of REM sleep. ${ }^{20,56} \mathrm{GiV}$ also sends inhibitory projections that inhibit REM sleep. ${ }^{36,57}$ However, how progressive Parkinsonism affects the REM sleep on/off switch still remains unclear.

Since the systems regulating sleep behaviors are not entirely known, other pathways may also be involved in regulating motor behavior in parasomnias, such as RBD and restless leg syndrome. ${ }^{58,59}$ Studies have found projections from globus pallidus externa to cortices, which regulate cortical activities during REM sleep. ${ }^{60,61}$ Direct projections from supra pontine structures to the spinal cord may also participate in disinhibited sleep behaviors. ${ }^{62,63}$ Therefore, the involvement of other motor regulatory pathways in early Parkinsonism needs further elucidation.

One finding of the present study is the progressive appearance of RBD symptoms that correlated well with pathological changes in SLD and GiV. Although this progression from SLD to GiV may not reflect actual changes in PD patients with prior RBD symptoms, it recapitulates some features of RBD development. As summarized and hypothesized in Figure 8, reduced frequency of brain rhythms was observed first, implicating reduced SLD projections to upstream brain regions, eg cortices. Later, as SLD neural activity is further impaired, dream enactments may be triggered. At this stage, RBD may associate with other sleep symptoms like sleep fragmentation and daytime sleepiness. Further pathological changes in GiV impaired spinal inhibition during all sleep types and increased behaviors or muscle twitches during NREM, along with all other RBD symptoms.

\section{Conclusions}

In conclusion, the chronic rotenone model of PD successfully recapitulates some key features of RBD. The mechanistic studies are consistent with progressive impairments in the pontine-medullary REM sleep promoting and atonia circuit in early Parkinsonism that underlies the emergence of RBD symptoms. Our results thus provide a foundation for further elucidation of PD-related RBD and early diagnosis of Parkinson's Disease.

\section{Abbreviations}

AAV, adeno-associated virus; CAMKII $\alpha$, calcium/calmodulin-dependent protein kinase II $\alpha$ chain; DAPI, 4',6-diamidino-2-phenylindole; DREADD, designer receptor exclusively activated by designer drugs; EEG, electroencephalography; EMG, electromyography; GABA, $\gamma$-aminobutyric acid; GAD67, glutamic acid decarboxylase 67; GiV, gigantocellular ventricular reticular nucleus; GPCR, G protein coupled receptor; NGS, normal goat serum; NREM, nonrapid eye movement; PD, Parkinson's disease; RBD, REM sleep behavior disorder; REM, rapid eye movement; SLD, sublaterodorsal tegmental nucleus; $\mathrm{TH}$, tyrosine hydroxylase; TUNEL, terminal deoxynucleotidyl transferase dUTP nick end labeling; vGluT2, vesicular glutamate transporter.

\section{Acknowledgments}

This work was supported by the Gerald Choa Neuroscience Centre 7105306 and the AoE grant of the Hong Kong Research Grants Council AoE/M-604/16.

All animal using procedures were approved by the Animal Experimentation Ethics Committee of the Chinese University of Hong Kong, following "International Guiding Principles for Biomedical Research Involving Animals", jointly published by the Council for International Organizations for Medical Science (CIOMS) and the International Council of Laboratory Animal Science (ICLAS).

\section{Disclosure}

Professor Yun-Kwok Wing reports personal fees from Eisai Co, and travel support from Lundbeck company, outside the submitted work. We declare no other conflict of interest in this work.

\section{References}

1. Schenck CH, Bundlie SR, Ettinger MG, Mahowald MW. Chronic behavioral disorders of human REM sleep: a new category of parasomnia. Sleep. 1986;9(2):293-308. doi:10.1093/sleep/9.2.293

2. Ohayon MM, Caulet M, Priest RG. Violent behavior during sleep. J Clin Psychiatry. 1997;58(8):369-376;quiz 377. doi:10.4088/JCP. v58n0808

3. Chiu HF, Wing YK, Lam LC, et al. Sleep-related injury in the elderlyan epidemiological study in Hong Kong. Sleep. 2000;23(4):513-517. doi:10.1093/sleep/23.4.1e

4. Bjørnarå KA, Dietrichs E, Toft M. REM sleep behavior disorder in Parkinson's disease-is there a gender difference? Parkinsonism Relat Disord. 2013;19(1):120-122. doi:10.1016/j.parkreldis.2012.05.027

5. Trotti LM, Bliwise DL. Treatment of the sleep disorders associated with Parkinson's disease. Neurotherapeutics. 2014;11(1):68-77. doi:10.1007/s13311-013-0236-z

6. Neikrug AB, Ancoli-Israel S. Diagnostic tools for REM sleep behavior disorder. Sleep Med Rev. 2012;16(5):415-429. doi:10.1016/j. smrv.2011.08.004

7. Sforza E, Krieger J, Petiau C. REM sleep behavior disorder: clinical and physiopathological findings. Sleep Med Rev. 1997;1(1):57-69. doi:10.1016/S1087-0792(97)90006-X 
8. Unger MM, Belke M, Menzler K, et al. Diffusion tensor imaging in idiopathic REM sleep behavior disorder reveals microstructural changes in the brainstem, substantia nigra, olfactory region, and other brain regions. Sleep. 2010;33(6):767-773. doi:10.1093/sleep/ 33.6.767

9. Boeve BF. REM sleep behavior disorder: updated review of the core features, the REM sleep behavior disorder-neurodegenerative disease association, evolving concepts, controversies, and future directions. Ann N Y Acad Sci. 2010;1184(1):15-54. doi:10.1111/j.17496632.2009.05115.x

10. Postuma RB, Gagnon JF, Pelletier A, Montplaisir J. Prodromal autonomic symptoms and signs in Parkinson's disease and dementia with Lewy bodies. Mov Disord. 2013;28(5):597-604. doi:10.1002/mds.25445

11. McCarter SJ, Tippmann-Peikert M, Sandness DJ, et al. Neuroimaging-evident lesional pathology associated with REM sleep behavior disorder. Sleep Med. 2015;16(12):1502-1510. doi:10.1016/j.sleep.2015.07.018

12. Iranzo A, Molinuevo JL, Santamaría J, et al. Rapid-eye-movement sleep behaviour disorder as an early marker for a neurodegenerative disorder: a descriptive study. Lancet Neurol. 2006;5(7):572-577. doi:10.1016/S1474-4422(06)70476-8

13. Schenck CH, Boeve BF, Mahowald MW. Delayed emergence of a parkinsonian disorder or dementia in $81 \%$ of older men initially diagnosed with idiopathic rapid eye movement sleep behavior disorder: a 16-year update on a previously reported series. Sleep Med. 2013;14(8):744-748. doi:10.1016/j.sleep.2012.10.009

14. Boeve BF, Silber MH, Saper CB, et al. Pathophysiology of REM sleep behaviour disorder and relevance to neurodegenerative disease. Brain. 2007;130(11):2770-2788. doi:10.1093/brain/awm056

15. Valencia Garcia S, Libourel PA, Lazarus M, Grassi D, Luppi PH, Fort P. Genetic inactivation of glutamate neurons in the rat sublaterodorsal tegmental nucleus recapitulates REM sleep behaviour disorder. Brain. 2017;140(2):414-428. doi:10.1093/brain/aww310

16. Park SH, Weber F. Neural and homeostatic regulation of REM sleep. Front Psychol. 2020;11:1662. doi:10.3389/FPSYG.2020.01662

17. Boissard R, Gervasoni D, Schmidt MH, Barbagli B, Fort P, Luppi PH. The rat ponto-medullary network responsible for paradoxical sleep onset and maintenance: a combined microinjection and functional neuroanatomical study. Eur $J$ Neurosci. 2002;16 (10):1959-1973. doi:10.1046/j.1460-9568.2002.02257.x

18. Chen MC, Yu H, Huang ZL, Lu J. Rapid eye movement sleep behavior disorder. Curr Opin Neurobiol. 2013;23(5):793-798. doi:10.1016/j.conb.2013.02.019

19. Peever J, Luppi PH, Montplaisir J. Breakdown in REM sleep circuitry underlies REM sleep behavior disorder. Trends Neurosci. 2014;37 (5):279-288. doi:10.1016/j.tins.2014.02.009

20. Lu J, Sherman D, Devor M, Saper CB. A putative flip-flop switch for control of REM sleep. Nature. 2006;441(7093):589-594. doi:10.1038/ nature 04767

21. Clément O, Sapin E, Bérod A, Fort P, Luppi P. Evidence that neurons of the sublaterodorsal tegmental nucleus triggering paradoxical (REM) sleep are glutamatergic. Sleep. 2011;34(4):419-423. doi:10.1093/sleep/ 34.4.419

22. Boeve BF, Silber MH, Ferman TJ, et al. Clinicopathologic correlations in 172 cases of rapid eye movement sleep behavior disorder with or without a coexisting neurologic disorder. Sleep Med. 2013;14 (8):754-762. doi:10.1016/j.sleep.2012.10.015

23. Iranzo A, Tolosa E, Gelpi E, et al. Neurodegenerative disease status and post-mortem pathology in idiopathic rapid-eye-movement sleep behaviour disorder: an observational cohort study. Lancet Neurol. 2013;12(5):443-453. doi:10.1016/S1474-4422(13)70056-5

24. Schenck CH, Bundlie SR, Mahowald MW. Delayed emergence of a parkinsonian disorder in $38 \%$ of 29 older men initially diagnosed with idiopathic rapid eye movement sleep behaviour disorder. Neurology. 1996;46(2):388-393. doi:10.1212/WNL.46.2.388
25. St Louis EK, Boeve AR, Boeve BF. REMSleep behavior disorder in Parkinson's disease and other synucleinopathies. Mov Disord. 2017;32(5):645-658. doi:10.1002/mds.27018

26. Braak H, Del Tredici K, Rüb U, de Vos RAI, Jansen Steur ENH, Braak E. Staging of brain pathology related to sporadic Parkinson's disease. Neurobiol Aging. 2003;24(2):197-211. doi:10.1016/S01974580(02)00065-9.

27. Gagnon JF, Postuma RB, Mazza S, Doyon J, Montplaisir J. Rapideye-movement sleep behaviour disorder and neurodegenerative diseases. Lancet Neurol. 2006;5(5):424-432. doi:10.1016/S14744422(06)70441-0

28. Petit D, Gagnon J-F, Fantini ML, Ferini-Strambi L, Montplaisir J. Sleep and quantitative EEG in neurodegenerative disorders. J Psychosom Res. 2004;56(5):487-496. doi:10.1016/j.jpsychores. 2004.02.001

29. Raggi A, Ferri R. Sleep disorders in neurodegenerative diseases. Eur $J$ Neurol. 2010;17(11):1326-1338. doi:10.1111/j.1468-1331.2010. 03034.x

30. Spillantini MG, Schmidt ML, Lee VMY, Trojanowski JQ, Jakes R, Goedert M. $\alpha$-synuclein in Lewy bodies. Nature. 1997;388 (6645):839-840. doi:10.1038/42166

31. Seidel K, Mahlke J, Siswanto S, et al. The brainstem pathologies of Parkinson's disease and dementia with Lewy bodies. Brain Pathol. 2015;25(2):121-135. doi:10.1111/bpa.12168

32. Barone DA, Henchcliffe C. Rapid eye movement sleep behavior disorder and the link to alpha-synucleinopathies. Clin Neurophysiol. 2018;129(8):1551-1564. doi:10.1016/j.clinph.2018.05.003

33. Betarbet R, Sherer TB, MacKenzie G, Garcia-Osuna M, Panov AV, Greenamyre JT. Chronic systemic pesticide exposure reproduces features of Parkinson's disease. Nat Neurosci. 2000;3 (12):1301-1306. doi:10.1038/81834

34. Hensley K, Pye QN, Maidt ML, et al. Interaction of $\alpha$-phenyl-N-tertbutyl nitrone and alternative electron acceptors with complex I indicates a substrate reduction site upstream from the rotenone binding site. J Neurochem. 2002;71(6):2549-2557. doi:10.1046/ j.1471-4159.1998.71062549.x

35. Ma C, Zhong P, Liu D, et al. Sleep regulation by neurotensinergic neurons in a thalamo-amygdala circuit. Neuron. 2019;103(2):323334.e7. doi:10.1016/j.neuron.2019.05.015

36. Weber F, Chung S, Beier KT, Xu M, Luo L, Dan Y. Control of REM sleep by ventral medulla GABAergic neurons. Nature. 2015;526 (7573):435-438. doi:10.1038/nature14979

37. Ravassard P, Pachoud B, Comte J, Mejia-perez C, Scoté-blachon C. Paradoxical (REM) sleep deprivation causes a large and rapidly reversible decrease in long-term potentiation, synaptic transmission, glutamate receptor protein levels, and ERK/MAPK activation in the dorsal hippocampus. Sleep. 2009;32(2):227-240. doi:10.1093/sleep/ 32.2.227

38. Li Y, Chopp M, Jiang N, Yao F, Zaloga C. Temporal profile of in situ DNA fragmentation after transient middle cerebral artery occlusion in the rat. $J$ Cereb Blood Flow Metab. 1995;15(3):389-397. doi:10.1038/jcbfm.1995.49

39. Livia fantini M, Gagnon JF, Petit D, et al. Slowing of electroencephalogram in rapid eye movement sleep behavior disorder. Ann Neurol. 2003;53(6):774-780. doi:10.1002/ana.10547

40. Gagnon JF, Fantini ML, Bédard MA, et al. Association between waking EEG slowing and REM sleep behavior disorder in PD without dementia. Neurology. 2004;62(3):401-406. doi:10.1212/01. WNL.0000106460.34682.E9

41. McCarley RW. Mechanisms and models of REM sleep control. Arch Ital Biol. 2004;142(4):429-467.

42. Anaclet C, Ferrari L, Arrigoni E, et al. The GABAergic parafacial zone is a medullary slow wave sleep-promoting center. Nat Neurosci. 2014;17(9):1217-1224. doi:10.1038/nn.3789

43. Weber F, Dan Y. Circuit-based interrogation of sleep control. Nature. 2016;538(7623):51-59. doi:10.1038/nature19773 
44. Fuller PM, Saper CB, Lu J. The pontine REM switch: past and present. J Physiol. 2007;584(Pt 3):735-741. doi:10.1113/ jphysiol.2007.140160

45. Massicotte-Marquez J, Decary A, Gagnon JF, et al. Executive dysfunction and memory impairment in idiopathic REM sleep behavior disorder. Neurology. 2008;70(15):1250-1257. doi:10.1212/01. wnl.0000286943.79593.a6

46. Nardone R, Bergmann J, Kunz A, et al. Cortical afferent inhibition is reduced in patients with idiopathic REM sleep behavior disorder and cognitive impairment: a TMS study. Sleep Med. 2012;13(7):919-925. doi:10.1016/j.sleep.2012.03.009

47. Caselli RJ, Chen K, Bandy D, et al. A preliminary fluorodeoxyglucose positron emission tomography study in healthy adults reporting dream-enactment behavior. Sleep. 2006;29(7):927-933. doi:10.1093/ sleep/29.7.927

48. Jones BE. Basic mechanisms of sleep-wake states. In: Principles and Practice of Sleep Medicine. Elsevier; 2005:136-153. doi:10.1016/B072-160797-7/50018-5

49. Richter F, Hamann M, Richter A. Chronic rotenone treatment induces behavioral effects but no pathological signs of parkinsonism in mice. J Neurosci Res. 2007;85(3):681-691. doi:10.1002/jnr.21159

50. Gureviciene I, Gurevicius K, Tanila H. Role of $\alpha$-synuclein in synaptic glutamate release. Neurobiol Dis. 2007;28(1):83-89. doi:10.1016/ j.nbd.2007.06.016

51. Day M, Wang Z, Ding J, et al. Selective elimination of glutamatergic synapses on striatopallidal neurons in Parkinson disease models. Nat Neurosci. 2006;9(2):251-259. doi:10.1038/nn1632

52. Pienaar IS, Elson JL, Racca C, Nelson G, Turnbull DM, Morris CM. Mitochondrial abnormality associates with type-specific neuronal loss and cell morphology changes in the pedunculopontine nucleus in Parkinson disease. Am J Pathol. 2013;183(6):1826-1840. doi:10.1016/j.ajpath.2013.09.002

53. Cox J, Pinto L, Dan Y. Calcium imaging of sleep-wake related neuronal activity in the dorsal pons. Nat Commun. 2016;7:10763. doi: $10.1038 /$ ncomms 10763
54. Schenkel E, Siegel JM. REM sleep without atonia after lesions of the medial medulla. Neurosci Lett. 1989;98:159-165. doi:10.1016/03043940(89)90503-X

55. Brooks PL, Peever JH. Impaired GABA and glycine transmission triggers cardinal features of rapid eye movement sleep behavior disorder in mice. J Neurosci. 2011;31(19):7111-7121. doi:10.1523/ JNEUROSCI.0347-11.2011

56. Saper CB, Fuller PM, Pedersen NP, Lu J, Scammell TE. Sleep state switching. Neuron. 2010;68(6):1023-1042. doi:10.1016/j. neuron.2010.11.032

57. Valencia Garcia S, Brischoux F, Clément O, et al. Ventromedial medulla inhibitory neuron inactivation induces REM sleep without atonia and REM sleep behavior disorder. Nat Commun. 2018;9 (1):504. doi:10.1038/s41467-017-02761-0

58. Jones R, Cavanna AE. The neurobiology and treatment of restless legs syndrome. Behav Neurol. 2013;26(4):283-292. doi:10.3233/ BEN-2012-120271

59. Högl B, Stefani A. REM sleep behavior disorder (RBD). Somnologie. 2017;21(S1):1-8. doi:10.1007/s11818-016-0048-6

60. Qiu M-H, Vetrivelan R, Fuller PM, Lu J. Basal ganglia control of sleep-wake behavior and cortical activation. Eur J Neurosci. 2010;31 (3):499-507. doi:10.1111/j.1460-9568.2009.07062.x

61. Lazarus M, Chen JF, Urade Y, Huang ZL. Role of the basal ganglia in the control of sleep and wakefulness. Curr Opin Neurobiol. 2013;23 (5):780-785. doi:10.1016/j.conb.2013.02.001

62. Hening WA. Restless legs syndrome: a sensorimotor disorder of sleep/wake motor regulation. Curr Neurol Neurosci Rep. 2002;2 (2):186-196. doi:10.1007/s11910-002-0029-y

63. Guo CN, Yang WJ, Zhan SQ, et al. Targeted disruption of supraspinal motor circuitry reveals a distributed network underlying Restless Legs Syndrome (RLS)-like movements in the rat. Sci Rep. 2017;7 (1):9905. doi:10.1038/s41598-017-10284-3
Nature and Science of Sleep

\section{Publish your work in this journal}

Nature and Science of Sleep is an international, peer-reviewed, open access journal covering all aspects of sleep science and sleep medicine, including the neurophysiology and functions of sleep, the genetics of sleep, sleep and society, biological rhythms, dreaming, sleep disorders and therapy, and strategies to optimize healthy sleep.
The manuscript management system is completely online and includes a very quick and fair peer-review system, which is all easy to use. Visit http://www.dovepress.com/testimonials.php to read real quotes from published authors. 